\title{
Endoscopic sphincterotomy to prevent post-ERCP pancreatitis in patients with biliary neoplasms: a multicenter retrospective cohort study
}

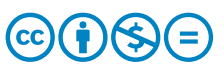

\author{
Authors \\ Institutions \\ 1 Second Department of Internal Medicine, Wakayama \\ Medical University, Wakayama, Japan \\ 2 Second Department of Internal Medicine, Osaka Medical \\ College, Osaka, Japan \\ 3 Department of Gastroenterology and Hepatology, \\ Kindai University Faculty of Medicine, Osaka, Japan \\ 4 Clinical Study Support Center, Wakayama Medical \\ University Hospital, Wakayama, Japan \\ 5 Second Department of Surgery, Wakayama Medical \\ University, Wakayama, Japan
}

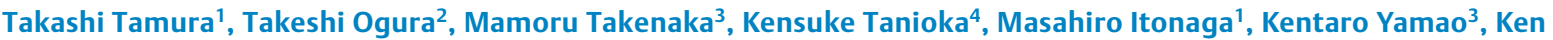

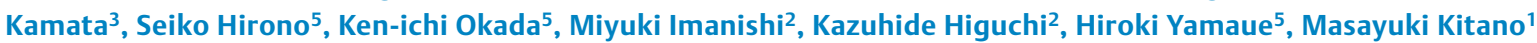

submitted 26.10.2018

accepted after revision 28.10.2019

Bibliography

DOI https://doi.org/10.1055/a-1107-2629 |

Endoscopy International Open 2020; 08: E513-E522

(c) Georg Thieme Verlag KG Stuttgart · New York

elSSN 2196-9736

Corresponding author

Masayuki Kitano, MD PhD, Second Department of Internal Medicine, Wakayama Medical University, 811-1, Kimiidera, Wakayama City, Wakayama 641-8509, Japan

Fax: +81-73-4453616

kitano@wakayama-med.ac.jp

Supplementary material

Online content viewable at:

https://doi.org/10.1055/a-1107-2629

\section{ABSTRACT}

Background and aims Treatment of biliary neoplasms often involves multiple endoscopic retrograde cholangiopancreatography (ERCP)-related procedures. Endoscopic sphincterotomy (ES) may prevent post-ERCP pancreatitis (PEP). This retrospective, multicenter cohort study aimed to investigate the effectiveness of ES for prevention of PEP in patients with biliary neoplasm.

Methods Patients with biliary duct neoplasm who underwent ERCP between January 2006 and December 2016 were enrolled. The frequency of PEP was compared between the ES and non-ES groups using propensity score analysis. The effectiveness of ES in subgroups of patients who underwent biliary duct stent placement, intraductal ultrasound (IDUS), and transpapillary biliary duct biopsy was analyzed by logistic regression.

Results Of the 362 patients enrolled, 84 (23.2\%) developed PEP. Propensity score matching for PEP risk factors in 172 ERCP procedures showed that the frequency of PEP in the ES group was lower than that in the non-ES group (19.7\% vs. $33.7 \%)$. Non-ES was also an independent risk factor for PEP in patients who underwent intraductal ultrasound and transpapillary biliary duct biopsy ( $R R=4.54$ and 5.26), but was not an independent risk factor for PEP in patients with biliary duct stents. In addition, there was no evidence that the frequency of PEP was statistically different between patients with plastic stents and metal stents in the ES and non-ES groups ( $P=0.14$ and 0.10$)$.

Conclusions ES is an effective technique to prevent PEP in patients with biliary neoplasms. In particular, ES is a safe technique to prevent PEP when performing IDUS and transpapillary biliary duct biopsy.

\section{Introduction}

Endoscopic retrograde cholangiopancreatography (ERCP) plays an important role in the inspection and treatment of biliary neoplasms. However, ERCP is an invasive treatment, and the frequency of post-ERCP pancreatitis (PEP) was reported to be $10-15 \%$ in patients with naïve papilla [1-4]. Several studies have shown that the demographic and clinical risk factors for PEP include younger age (<60 years), female sex, sphincter of Oddi dysfunction, a history of recurrent pancreatitis, and a history of PEP. Procedure-related risk factors for PEP include endoscopic papillary balloon dilation, pancreatic sphincterotomy, precut sphincterotomy, pancreatic duct injection, and repeated cannulation attempts [3,5-7]. However, few studies have 
described the risk factors for PEP in specific biliary diseases, which may be important because ERCP-related procedures vary depending on the disease. This variability in ERCP-related procedures is particularly pronounced in the treatment of biliary neoplasms. Multiple ERCP-related procedures, such as endoscopic biliary drainage (EBD), intraductal ultrasound (IDUS), and transpapillary biliary duct biopsy, are often also performed when treating biliary neoplasm. It is important to further clarify the method in terms of preventing PEP in patients with biliary neoplasm because these patients may undergo surgery to remove the neoplasm after ERCP and such surgery will be complicated if PEP develops.

Several studies report preventative methods for PEP, including the administration of nonsteroidal anti-inflammatory drugs (NSAIDs) before ERCP, pancreatic duct stenting, and wire-guided cannulation [8-13]. In addition, some endoscopists perform endoscopic sphincterotomy (ES) before biliary duct stent placement, IDUS, or transpapillary biliary duct biopsy because ES reduces tension at the pancreatic duct orifice and is therefore thought to help prevent PEP [14]. A recent meta-analysis also demonstrated that ES was associated with a lower risk of PEP in patients undergoing biliary stenting for bile leak [12].

This retrospective, multicenter study aimed to clarify the effectiveness of ES in the prevention of PEP in patients treated for biliary neoplasms. In cases of ES performed in patients with biliary neoplasm, the use of techniques such as biliary duct stent placement, IDUS, and/or transpapillary biliary duct biopsy may introduce bias regarding technical risk factors for PEP. We therefore undertook a propensity score analysis, which can balance the effects of many confounding risk factors.

\section{Materials and methods}

This retrospective, multicenter cohort study was approved by the institutional review boards of the three participating university hospitals.

\section{Patients}

The cohort consisted of all consecutive patients with biliary tract tumors who underwent ERCP for the first time between January 2006 and December 2016 at three tertiary care medical centers in Japan (Wakayama Medical University, Kindai University, and Osaka Medical University). Patients were excluded from the study if they were $<20$ years old, had a history of acute pancreatitis, and/or had previously undergone gastrectomy, $\mathrm{EBD}$, and/or ES.

\section{ERCP procedures and post-procedural care}

All patients provided informed consent before ERCP. All procedures were conducted by endoscopists who had performed> 200 ERCPs in total before this study. ERCP was performed according to a standard technique under intravenous sedation. In cases where cannulation was difficult, precut sphincterotomy or the double-wire technique was performed. After successful selective cannulation, cholangiography was performed to verify the biliary neoplasm. In addition, all patients received protease inhibitors. ERCP-related procedures (biliary duct stent placement, IDUS, and/or transpapillary biliary duct biopsy) were conducted to inspect the biliary neoplasm and treat the biliary obstruction. ES was performed using an electrosurgical generator in 120 Watt ENDO CUT mode with a papillotomy knife (CleverCut, Olympus Medical Systems) in all patients. The incision range was small (incision reached the hooding fold) or medium (incision reached up to a portion of the hooding fold) in all patients. Biliary duct stent placement was performed using a plastic stent (PS), a metal stent (MS), or an endoscopic naso-biliary drainage tube. The type of biliary stent was decided by the endoscopist, but a MS was only used in patients with inoperable cancer. MSs were uncovered or fully covered with a diameter of $10 \mathrm{~mm}$. Covered MSs were only placed across the papilla with ES. IDUS was conducted using a 3D-IDUS probe (UM-DG20-31 R, Olympus Medical Systems). Transpapillary biliary duct biopsy was conducted using biopsy forceps (Radial Jaw 3GP/4P, Boston Scientific, or FB-38W or FB19N-1, Olympus Medical Systems). Biliary biopsy with forceps was performed under X-ray fluoroscopy using the following four techniques according to previous reports $[15,16]$ : direct insertion of forceps with the free-hand technique without ES, wire-guided insertion of forceps with a side slit for the guidewire without ES, wire-guided insertion of forceps with grasping the guidewire without ES, and direct insertion of forceps with the freehand technique with ES. Pancreatic duct stents were placed at the discretion of the endoscopist $[15,16]$. Transpapillary biliary duct biopsy was performed 3-4 times.

On the first day after treatment, all patients underwent a physical examination and blood testing. When PEP occurred, the patients were treated immediately. Complications arising post-ERCP were recorded as early (in the first week) or late (more than 1 week after ERCP).

\section{Data collection}

The following baseline demographic and clinical data were obtained from the medical records: age, sex, body mass index, type and location of biliary neoplasm, and presence of common bile duct dilatation ( $\geq 8 \mathrm{~mm}$ ), peripapillary diverticulum, obstructive jaundice, and cholangitis. The bile duct diameter was defined as the common bile duct diameter on computed tomography, endoscopic ultrasound, or magnetic resonance cholangiopancreatography. When the neoplasm was in the common bile duct, measurement on the liver side was taken as the bile duct diameter.

The following procedural characteristics were also obtained from the medical records: total procedural time (from endoscope insertion to endoscope removal), cannulation method (whether wire-guided cannulation was used), and whether ES, biliary duct stent placement, IDUS, transpapillary biliary duct biopsy, pancreatic duct injection, double-wire technique, precut sphincterotomy, and pancreatic duct stent placement were performed. In addition, the use of NSAIDs was recorded and whether cholecystectomy was performed and if the tumor was treated operatively. Cannulation success was recorded along with the clinical symptoms and serum amylase levels 18-24 hours after the procedure. 


\section{Outcomes}

The outcome was the frequency of PEP. PEP was considered to be present when the patient experienced abdominal pain for $\geq 24$ hours after ERCP and had high serum amylase levels (three times the upper limit of normal) 18-24 hours after the procedure [17]. Another end point was effectiveness of ES for preventing PEP in subgroups. Biliary stent placement (PS placement, MS placement and MS placement across the papilla), IDUS, and transpapillary biliary duct biopsy are the final purposes of ERCP, particularly for biliary neoplasm and were therefore selected for subgroup analysis to evaluate the effectiveness of ES for preventing PEP. However, other technical risk factors, such as pancreatic duct injection, wire-guided cannulation, double guidewire technique, and precut, were performed to accomplish these purposes. Therefore, pancreatic duct injection, wire-guided cannulation, double guidewire technique, and precut were not selected as subgroups for analysis. The frequency of PEP in each group (ES and non-ES) was compared between patients with PSs and MSs. Other end points were the frequency of severe PEP, duration of the fasting period required to treat PEP, and the frequency of non-PEP complications after ERCP (perforation, biliary infection, bleeding, and any complications that required hospital treatment). PEP severity was classified according to Cotton's criteria [17]: mild (requiring 2-3 days of hospital treatment), moderate (4-10 days of hospital treatment), or severe (>10 days of hospital treatment, requiring surgical or intensive treatment, or where PEP contributed to the death of the patient) [14].

\section{Statistical analysis}

Demographic, pre-ERCP clinical, and ERCP procedural factors were compared between the ES and non-ES groups using the Chi-squared test. Propensity score matching analysis was performed. The propensity score calculated for each patient was based on logistic regression analysis of factors considered to affect PEP. These factors were selected using a forward stepwise method because many factors affect PEP. Using these propensity scores, cases and controls were matched one-to-one [1820]. Specifically, one-to-one matching was performed for the ES and non-ES groups ( $\triangleright$ Fig. 1) using nearest neighbor matching with a caliper coefficient of 0.2 . After matching, the incidence of PEP was compared between the matched ES and nonES groups.

In the five subgroups of patients who underwent biliary duct stent placement, PS placement, MS placement, IDUS, and transpapillary biliary duct biopsy, pre-ERCP clinical and ERCP procedural data were compared in patients with and without PEP by Chi-squared test. These univariate analyses were performed on data from the subgroups. Log binomial regression analysis was then used to identify factors related to PEP in the subgroups. In these subgroup analyses, propensity score matching was inappropriate because the number of matched pairs was small. Factors were included in each regression analysis if the $P$ value of the univariate test for that variable was< 0.10 ; this $P$ value was selected because of the exploratory nature of the study. Precut sphincterotomy and NSAID use were

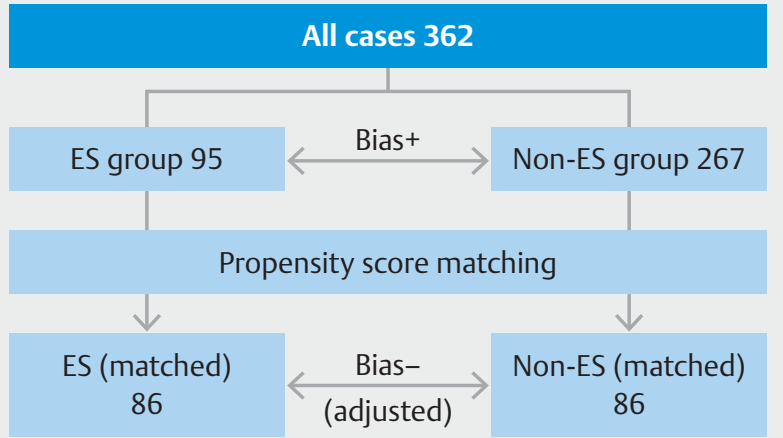

- Fig. 1 Schematic representation of propensity score matching analysis. In an effort to balance the patient group, propensity score analysis was used to generate a set of matched cases and controls. The propensity score was calculated for 362 patients based on a logistic analysis of clinical characteristics indicated by stepwise regression analysis (e. g. wire-guided cannulation, placement of biliary stent, NSAID administration, and transpapillary biliary duct biopsy). Using propensity scores, 86 patients were selected from 95 patients undergoing $E S$, and 86 patients from 267 patients in the non-ES group. ES, endoscopic sphincterotomy; NSAID, nonsteroidal anti-inflammatory drug.

not included in the subgroup regression analyses because the numbers of patients who underwent these treatments were too small. ES was included in all regression analyses irrespective of statistical significance on univariate analysis in order to assess its ability to predict PEP. In the subgroups of patients who underwent biliary duct MS placement across the papilla, preERCP clinical and ERCP procedural data were compared between patients with and without PEP using Fisher's exact test. The frequency of PEP in each group (ES and non-ES) was compared between patients with PSs and MSs using the Chisquared test.

All statistical analyses were performed using JMP (Version Pro 13.0) and R (version 3.4.1) software. For all analyses, $P$ values of $<0.05$ were considered to indicate statistical significance.

\section{Results}

In total, 362 patients with a mean age of 71.0 years were enrolled in the study. Of these, $12.9 \%$ were $<60$ years old and $46.2 \%$ were female; $16.8 \%$ and $4.4 \%$ had a body mass index $>25 \mathrm{~kg} / \mathrm{m}^{2}$ and a history of cholecystectomy, respectively. In addition, $50.4 \%, 14.0 \%, 17.9 \%, 2.2 \%$, and $5.5 \%$ had extrahepatic cholangiocarcinoma, papillary carcinoma, hilar cholangiocarcinoma, intrahepatic cholangiocarcinoma, and gallbladder carcinoma, respectively ( $>$ Table 1 ). At the time of ERCP, 63.9\%, $57.3 \%$, $12.9 \%$, and $8.8 \%$ had common bile duct dilatation, jaundice, cholangitis, and periampullary diverticulum, respectively. The mean total ERCP procedural time was 29.9 minutes; additional procedures were wire-guided cannulation (65.5\%), ES (26.2\%), biliary duct stent placement $(84.0 \%)$, IDUS (31.8\%), transpapillary biliary duct biopsy $(23.6 \%)$, pancreatic duct injection 
- Table 1 Demographic, pre-ERCP clinical, and ERCP procedural characteristics of the whole cohort of patients with biliary neoplasms $(n=362)$.

\begin{tabular}{|c|c|}
\hline Variables & n (\%) \\
\hline Younger age, $<60$ years & $47(12.9)$ \\
\hline Female sex & $168(46.2)$ \\
\hline Body mass index $>25 \mathrm{~kg} / \mathrm{m}^{2}$ & $61(16.8)$ \\
\hline \multicolumn{2}{|l|}{ Biliary tumor type } \\
\hline - Extrahepatic cholangiocarcinoma & $187(50.4)$ \\
\hline - Papillary carcinoma & $51(14.0)$ \\
\hline - Hilar cholangiocarcinoma & $65(17.9)$ \\
\hline - Intrahepatic cholangiocarcinoma & $8(2.2)$ \\
\hline - Gallbladder carcinoma & $20(5.5)$ \\
\hline - Others & $31(8.5)$ \\
\hline Common bile duct dilatation, $\geq 8 \mathrm{~mm}$ & $232(63.9)$ \\
\hline Periampullary diverticulum & $32(8.8)$ \\
\hline Jaundice at ERCP & $208(57.3)$ \\
\hline Pancreatic duct obstruction & $3(0.8)$ \\
\hline History of cholecystectomy & $16(4.4)$ \\
\hline Cholangitis & $47(12.9)$ \\
\hline Operative treatment on biliary duct tumor & $117(32.2)$ \\
\hline Cannulation success & $352(97.2)$ \\
\hline Median total procedural time, minutes & 29.9 \\
\hline Underwent wire-guided cannulation & $237(65.5)$ \\
\hline ES & $95(26.2)$ \\
\hline - Small incision range & $14(3.9)$ \\
\hline - Medium incision range & $81(22.3)$ \\
\hline Biliary duct stent placement & $304(84.0)$ \\
\hline \multicolumn{2}{|l|}{ Biliary duct stent type } \\
\hline - Metal stent & $68(19.5)$ \\
\hline - ENBD tube & $93(25.6)$ \\
\hline - Plastic stent & $143(38.6)$ \\
\hline Stent placement across the papilla & $260(71.8)$ \\
\hline IDUS & $115(31.8)$ \\
\hline Transpapillary biliary duct biopsy & $85(23.6)$ \\
\hline Pancreatic duct injection & $165(45.6)$ \\
\hline Double guidewire technique & $53(14.6)$ \\
\hline Precut sphincterotomy & $13(3.6)$ \\
\hline Pancreatic duct stent placement & $18(5.0)$ \\
\hline Administered NSAIDs & $17(4.7)$ \\
\hline
\end{tabular}

- Table 2 Complications associated with ERCP in patients with biliary neoplasm $(n=362)$.

\begin{tabular}{|c|c|}
\hline Variables & n (\%) \\
\hline Overall & $92(25.4)$ \\
\hline \multicolumn{2}{|c|}{ Early complications ${ }^{1}$} \\
\hline - Pancreatitis ${ }^{2}$ & $84(23.2)$ \\
\hline - Mild & $61(16.8)$ \\
\hline - Moderate & $16(4.4)$ \\
\hline - Severe & $7(1.9)$ \\
\hline - Bleeding & $3(0.8)$ \\
\hline - Cholangitis & $2(0.5)$ \\
\hline \multicolumn{2}{|c|}{ Late complications ${ }^{3}$} \\
\hline - Cholangitis & $3(0.8)$ \\
\hline \multicolumn{2}{|c|}{$\begin{array}{l}\text { ERCP, endoscopic retrograde cholangiopancreatography. } \\
{ }^{1} \text { Complications in the first } 7 \text { days after ERCP. } \\
2 \text { PEP severity was classified according to Cotton's criteria [14]. } \\
3 \text { Complications arising }>7 \text { days after ERCP. }\end{array}$} \\
\hline
\end{tabular}

(45.6\%), double guidewire technique (14.6\%), precut sphincterotomy (3.6\%), and pancreatic duct stent placement (5.0\%). In addition, $4.7 \%$ received NSAIDs and $32.2 \%$ underwent operative treatment on the biliary duct tumor.

\section{Complications associated with ERCP}

PEP occurred in 84 patients (23.2\%). It was mild, moderate, and severe in 61 (16.8\%), $16(4.4 \%)$, and 7 patients (1.9\%), respectively ( $\triangleright$ Table 2 ). The mean fasting period required to treat PEP was 4.97 days. Soon after ERCP, three $(0.8 \%)$ and two $(0.5 \%)$ patients developed bleeding and biliary infection, respectively. Three $(0.8 \%)$ developed cholangitis as a late post-ERCP complication.

\section{Patient characteristics in the ES versus non-ES group}

The characteristics of the ES and non-ES groups are shown in - Table 3. Differences were seen between the groups in the number of patients experiencing a long procedural time and the number undergoing wire-guided cannulation, IDUS, transpapillary biliary duct biopsy, pancreatic duct injection, double guidewire technique, precut sphincterotomy, and biliary duct stent placement. NSAID administration differed between the two groups.

\section{PEP in the ES versus non-ES group}

To minimize the effect of selection bias between the two groups, we performed propensity score matching analysis based on risk factors (cannulation method type, transpapillary biliary duct biopsy, biliary duct stent placement, and NSAIDs), which were chosen by a stepwise method, and selected 86 matched cases in each group in a one-to-one manner.

The C-statistic calculated from the receiver operating characteristic curve of our model was 0.74 , showing that our model had a good ability to distinguish ES patients from non-ES pa- 
- Table 3 Patient characteristics.

\begin{tabular}{|c|c|c|c|c|c|c|}
\hline \multirow[t]{2}{*}{ Variables } & \multicolumn{3}{|c|}{ All patients } & \multicolumn{3}{|c|}{ Patients selected by propensity matching } \\
\hline & $\begin{array}{l}\text { ES group } \\
n=95\end{array}$ & $\begin{array}{l}\text { Non-ES group } \\
n=267\end{array}$ & $P$ value $^{1}$ & $\begin{array}{l}\text { ES group after } \\
\text { matching } \\
n=86\end{array}$ & $\begin{array}{l}\text { Non-ES group } \\
\text { after matching } \\
n=86\end{array}$ & $P$ value $^{1}$ \\
\hline Younger age, $<60$ years & $9(9.5)$ & $38(14.2)$ & 0.23 & $7(8.1)$ & $13(15.2)$ & 0.15 \\
\hline Female sex & $44(46.4)$ & $124(46.4)$ & 0.98 & $38(44.2)$ & $34(39.5)$ & 0.53 \\
\hline Body mass index $>25 \mathrm{~kg} / \mathrm{m}^{2}$ & $18(19.0)$ & $43(16.2)$ & 0.53 & $17(19.8)$ & $17(19.8)$ & 1.00 \\
\hline \multicolumn{7}{|l|}{ Biliary tumor location } \\
\hline - Papilla & $8(8.4)$ & $44(16.5)$ & 0.05 & $7(8.1)$ & $15(17.4)$ & 0.07 \\
\hline - Hilar bile duct & $17(18.0)$ & $56(21.0)$ & 0.52 & $15(17.4)$ & $13(15.1)$ & 0.68 \\
\hline - Extrahepatic bile duct & $70(73.7)$ & $167(62.7)$ & 0.05 & $64(74.4)$ & $58(67.4)$ & 0.31 \\
\hline Common bile duct diameter $<8 \mathrm{~mm}$ & $34(35.8)$ & $96(36.0)$ & 0.98 & $29(33.7)$ & $25(29.1)$ & 0.51 \\
\hline Periampullary diverticulum & $3(3.2)$ & $29(10.9)$ & 0.02 & $3(3.5)$ & $4(4.7)$ & 0.70 \\
\hline Jaundice at ERCP & $61(64.2)$ & $147(55.1)$ & 0.12 & $56(65.1)$ & $52(60.5)$ & 0.53 \\
\hline Long procedural time, $>40 \mathrm{~min}$ & $48(50.5)$ & $89(33.3)$ & $0.003^{1}$ & $43(50.0)$ & $32(37.2)$ & 0.09 \\
\hline Wire-guided cannulation & $84(88.4)$ & $157(57.3)$ & $<0.001^{1}$ & $75(87.2)$ & $75(87.2)$ & 1.00 \\
\hline IDUS & $42(41.2)$ & $73(27.3)$ & $0.002^{1}$ & $38(44.2)$ & $30(34.9)$ & 0.21 \\
\hline Transpapillary biliary duct biopsy & $34(35.8)$ & $51(19.2)$ & $0.001^{1}$ & $30(34.9)$ & $30(34.9)$ & 1.00 \\
\hline Pancreatic duct injection & $52(54.7)$ & $113(42.3)$ & $0.04^{1}$ & $46(53.5)$ & $47(54.7)$ & 0.87 \\
\hline Double guidewire technique & $21(22.1)$ & $32(12.0)$ & $0.02^{1}$ & $17(19.8)$ & $17(19.8)$ & 1.00 \\
\hline Precut sphincterotomy & $7(7.4)$ & $6(2.3)$ & $0.02^{1}$ & $5(5.8)$ & $2(2.5)$ & 0.25 \\
\hline Pancreatic duct stent placement & $5(5.3)$ & $13(4.9)$ & 0.88 & $4(4.7)$ & $5(5.8)$ & 0.73 \\
\hline Biliary duct stent placement & $87(91.6)$ & $217(81.3)$ & $0.02^{1}$ & $78(90.7)$ & $78(90.7)$ & 1.00 \\
\hline NSAID administration & $11(11.6)$ & $6(2.3)$ & $0.0002^{1}$ & $4(4.7)$ & $4(4.7)$ & 1.00 \\
\hline $\begin{array}{l}\text { ERCP, endoscopic retrograde cholangiop } \\
\text { trasound; NSAIDs, nonsteroidal anti-infla } \\
\text { The data are shown as } n(\%) \text {. } P \text { values we } \\
1 P \text { values }<0.05 \text { (bold) were considered }\end{array}$ & $\begin{array}{l}\text { reatography; } \\
\text { natory drugs. } \\
\text { alculated usi } \\
\text { e significant. }\end{array}$ & $\begin{array}{l}\text {, endoscopic sphin } \\
\text { Chi-squared test. }\end{array}$ & erotomy not $\mathrm{p}$ & ormed; ES, endoscor & hincterotomy; IDUS & ductal ul- \\
\hline
\end{tabular}

tients. After matching, there was no evidence for differences between in patient clinical characteristics or procedural characteristics in the two groups, apart from the statistically significant difference in the risk factors for PEP ( $>$ Table 3 ). The incidence of PEP in the matched ES group was lower than that in the non-ES group (19.7\% vs. $33.7 \%, P=0.04$; Odds Ratio (OR) $=0.47,95 \%$ Confidence interval $(\mathrm{Cl}): 0.23-0.96)$. The incidence of each severity of PEP and other complications did not differ between the two groups ( $\triangleright$ Table 4 ).

\section{Frequency of PEP according to the stent type (PSs versus MSs)}

There were no evidence for differences in the frequency of PEP between patients with PSs and MSs in the ES group (16.7\% vs. $30.3 \%, P=0.14, \mathrm{OR}=0.46,95 \% \mathrm{Cl}: 0.17-1.26)$ and the non-ES group $(21.4 \%$ vs. $34.3 \%, P=0.10, O R=0.52,95 \% \mathrm{Cl}: 0.24-$ 1.13) ( Table 5).

\section{Frequency of PEP according to the incision range of ES (small versus medium)}

There was no evidence for differences in the frequency of PEP between patients with small and medium incision ranges (28.6\% vs. $21.0 \%$; $P=0.53, \mathrm{OR}=0.64,95 \% \mathrm{Cl} 0.19-2.38$ ).

\section{Risk factors for PEP in patients undergoing biliary duct stent placement, PS placement, MS placement, IDUS, and transpapillary biliary duct biopsy}

Univariable and multivariable analyses in the 304 patients who underwent biliary duct stent placement showed that jaundice at ERCP $(R R=1.60 ; P=0.03)$, pancreatic duct injection $(R R=$ 2.01; $P=0.002$ ), and double guidewire cannulation ( $R R=1.54$; $P=0.04)$ were considered to be independent risk factors for PEP ( Table 6). Univariable and multivariable analyses of patients with PSs showed that non-ES was a risk factor for PEP $(R R=1.95 ; P=0.03)$ (Supplementary table 1$)$. Univariable and 
- Table 4 Complications associated with ERCP in ES and non-ES groups after propensity score matching.

\begin{tabular}{|c|c|c|c|c|c|}
\hline Variables & $\begin{array}{l}\text { ES group after matching } \\
n=86\end{array}$ & $\begin{array}{l}\text { Non-ES group after matching } \\
n=86\end{array}$ & $P$ value & OR & $95 \% \mathrm{Cl}$ \\
\hline PEP & $17(19.7)$ & $29(33.7)$ & $0.04^{1}$ & 0.47 & $0.23-0.96$ \\
\hline - Mild & $15(17.4)$ & $23(26.7)$ & 0.14 & 0.57 & $0.28-1.20$ \\
\hline - Moderate & $1(1.1)$ & $2(2.3)$ & 0.56 & 0.49 & $0.06-3.86$ \\
\hline - Severe & $1(1.1)$ & $4(4.7)$ & 0.17 & 0.24 & $0.04-1.65$ \\
\hline Bleeding & $3(3.4)$ & $0(0)$ & 0.24 & $\operatorname{lnf}$ & $\operatorname{lnf}$ \\
\hline Cholangitis & $1(1.0)$ & $0(0)$ & 1.00 & $\operatorname{lnf}$ & $\operatorname{lnf}$ \\
\hline
\end{tabular}

- Table 5 Frequency of PEP in patients with plastic and metal stents.

\begin{tabular}{|c|c|c|c|c|c|c|c|c|c|c|}
\hline \multirow[t]{2}{*}{ Variable } & \multicolumn{5}{|l|}{$\begin{array}{l}\text { ES group } \\
n=87\end{array}$} & \multicolumn{5}{|c|}{$\begin{array}{l}\text { Non-ES group } \\
n=217\end{array}$} \\
\hline & $\begin{array}{l}\text { Plastic } \\
\text { stent } \\
\mathrm{n}=\mathbf{5 4}\end{array}$ & $\begin{array}{l}\text { Metal } \\
\text { stent } \\
n=33\end{array}$ & $P$ value & OR & $95 \% \mathrm{Cl}$ & $\begin{array}{l}\text { Plastic } \\
\text { stent } \\
n=182\end{array}$ & $\begin{array}{l}\text { Metal } \\
\text { stent } \\
n=35\end{array}$ & $P$ value & OR & $95 \% \mathrm{Cl}$ \\
\hline PEP & $9(16.7 \%)$ & $10(30.3 \%)$ & 0.14 & 0.46 & $0.17-1.26$ & $39(21.4 \%)$ & 12 (34.3\%) & 0.10 & 0.52 & $0.24-1$. \\
\hline
\end{tabular}

OR, odds ratio; $\mathrm{Cl}$, Confidence interval; PEP, post-ERCP pancreatitis; non-ES, endoscopic sphincterotomy not performed; ES, endoscopic sphincterotomy.

multivariable analyses of patients with MSs showed that, statistically, there was no evidence that non-ES was a risk factor for PEP. In addition, there was no evidence that the number of patients without ES differed between the PEP and non-PEP groups among patients with MS placement across the papilla (Supplementary tables 2 and 3). However, the number of patients without ES was higher in the PEP group than in the non-PEP group among patients without biliary duct stent placement $(P=0.03)$ (Supplementary table 4).

Univariable and multivariable analyses of the 115 patients who underwent IDUS showed that non-ES $(R R=4.54 ; P=0.01)$ and a long procedural time $(R R=2.89 ; P=0.01$; Table 7$)$ were statistically significant risk factors for PEP.

Univariable and multivariable analyses of the 85 patients who underwent transpapillary biliary duct biopsy showed that female sex $(R R=5.25 ; P=0.03)$ and non-ES (RR=5.26; $P=$ 0.017 ) were risk factors for PEP in this group of patients ( $\triangle$ Table 8$)$.

\section{Discussion}

In this study, $23.2 \%$ of patients who underwent ERCP for biliary duct tumors developed PEP; the rate of severe PEP was $1.9 \%$. This incidence of PEP and severe PEP is higher than that reported in previous studies (5-15\% and $0.3-1.0 \%$, respectively) and may reflect the fact that the current study focused on biliary duct tumors with naive papilla, whereas previous studies examined PEP in patients with a variety of diseases [1-4]. After per- forming propensity score matching to minimize the effect of selection bias between the groups, the frequency of PEP was lower in the ES group than in the non-ES group. This study suggests that ES is an effective method for preventing PEP in patients with biliary duct neoplasm.

$E S$ is a common and often essential procedure in therapeutic ERCP. In the present study, PEP risk factors were more commonly seen in the ES group, which may influence the outcome and mask the true effectiveness of ES. Therefore, propensity score matching analysis was undertaken to minimize the effects of any inherent bias and to reduce the effects of confounding factors identified in observational studies $[18,20$, 21]. Recent studies reported that treatment effects from randomized trials and propensity score analysis were similar in similar populations [22]. In this study, matching effectively eliminated differences in clinical characteristics between the ES and non-ES groups, including the risk factors for PEP. After matching, ES decreased the incidence of PEP. However, after propensity score matching, the incidence of PEP in the non-ES group (33.7\%) was higher than previously reported [2-4]. This may be because the percentage of patients with a risk factor for PEP increased after propensity score matching.

Some previous studies have reported that ES increases the frequency of PEP. For example, a large case series conducted by Rabenstein et al. (2000) showed ES to be associated with a high rate of PEP [23]. Akashi et al. (2002) reported that this association reflects the sensitivity of the pancreatic duct to ES-induced thermal damage, which generates edema in the sur- 
- Table 6 Risk factors for PEP in patients who underwent biliary duct stent placement.

\begin{tabular}{|c|c|c|c|c|c|}
\hline \multirow[t]{2}{*}{ Variables } & \multicolumn{3}{|c|}{ Univariable analysis ${ }^{1}$} & \multicolumn{2}{|c|}{ Multivariable analysis } \\
\hline & $\begin{array}{l}\text { PEP } \\
n=70\end{array}$ & $\begin{array}{l}\text { No PEP } \\
n=234\end{array}$ & $P$ value $^{1}$ & $\mathbf{R R}$ & $P$ value \\
\hline Younger age, $<60$ years & $10(14.2)$ & $31(13.2)$ & 0.82 & & \\
\hline Female sex & $35(50.0)$ & $100(42.7)$ & 0.28 & & \\
\hline Body mass index $>25 \mathrm{~kg} / \mathrm{m}^{2}$ & $11(15.7)$ & $33(14.1)$ & 0.70 & & \\
\hline \multicolumn{6}{|l|}{ Biliary location of tumor } \\
\hline - Papilla & $9(12.8)$ & $35(14.9)$ & 0.67 & & \\
\hline - Hilar bile duct & $17(24.2)$ & $42(17.9)$ & 0.56 & & \\
\hline - Extrahepatic bile duct & $44(62.8)$ & $157(67.1)$ & 0.63 & & \\
\hline Common bile duct diameter $<8 \mathrm{~mm}$ & $26(37.1)$ & $75(32.0)$ & 0.42 & & \\
\hline Periampullary diverticulum & $8(11.4)$ & $17(7.3)$ & 0.27 & & \\
\hline Jaundice at ERCP & $51(72.8)$ & $134(49.6)$ & $0.03^{1}$ & 1.60 & $0.03^{1}$ \\
\hline Long procedural time, $>40 \mathrm{~min}$ & $33(47.1)$ & $81(34.6)$ & 0.06 & 1.38 & 0.10 \\
\hline Cannulation method type ${ }^{2}$ & $19(27.1)$ & $84(35.9)$ & 0.17 & & \\
\hline Non-ES & $49(70.0)$ & $168(71.8)$ & 0.77 & 1.05 & 0.81 \\
\hline IDUS & $16(22.9)$ & $84(35.9)$ & $0.04^{1}$ & 0.51 & $0.01^{1}$ \\
\hline Placement metal stent & $23(32.9)$ & $48(20.5)$ & $0.04^{1}$ & 0.91 & 0.64 \\
\hline Stent placement across the papilla & $58(82.8)$ & $202(86.3)$ & 0.47 & & \\
\hline Transpapillary biliary duct biopsy & $14(20.0)$ & $54(23.1)$ & 0.73 & & \\
\hline Pancreatic duct injection & $43(61.4)$ & $92(39.3)$ & 0.001 & 2.01 & $0.002^{1}$ \\
\hline Double guidewire technique & $21(30.0)$ & $25(10.7)$ & 0.001 & 1.54 & $0.04^{1}$ \\
\hline Pancreatic duct stent placement & $1(1.4)$ & $14(6.0)$ & 0.21 & & \\
\hline \multicolumn{6}{|c|}{$\begin{array}{l}\text { ERCP, endoscopic retrograde cholangiopancreatography; non-ES, endoscopic sphincterotomy not performed; IDUS, intraductal ultrasound; RR, Relative risk; PEP, } \\
\text { post-ERCP pancreatitis. } \\
\text { The data are shown as } n \text { (\%). } \\
{ }^{1} \text { Univariable analyses were performed using Chi-squared tests. P values of }<0.05 \text { (bold) were considered to be sufficiently significant for inclusion in the logistic } \\
\text { regression analysis. } \\
{ }^{2} \text { Not guided by wire. }\end{array}$} \\
\hline
\end{tabular}

rounding tissues and temporarily blocks the pancreatic duct [24]. The retrospective cohort study by Kawakubo et al. (2012) reported that, in patients with malignant biliary obstruction, those who did not undergo ES were not at increased risk of PEP after transpapillary biliary stent placement [25]. However, several other studies demonstrated a protective effect associated with ES in patients undergoing biliary stenting. First, the randomized controlled trial by Zhou et al. (2012) showed that not conducting ES before intraductal placement of self-expanding MSs increased the rate of PEP in patients with malignant obstructive biliary disease [26]. Sofi et al. (2016) reported that ES before biliary stenting is protective against PEP in patients with bile leak [12]. Therefore, whether ES is protective or actually increases the risk of PEP remains controversial.

Our subgroup analyses showed that, in patients with placement of biliary duct stent, injection of contrast agent into the pancreatic duct and double guidewire technique were indepen- dent risk factors for PEP. Non-ES, however, was not an independent risk factor for PEP in these patients. In addition, ES did not effectively prevent PEP in patients with MSs or even in patients who underwent MS placement across the papilla. In contrast, non-ES was seen to be an independent risk factor for PEP in patients who underwent PS placement, IDUS, and transpapillary biopsy. In addition, ES did not effectively prevent PEP in patients without biliary duct stent placement. This suggests that ES is more effective in the prevention of PEP in patients undergoing PS placement, IDUS and transpapillary biopsy than in patients undergoing MS placement. It is reported that ES can lower the risk of PEP by reducing tension at the pancreatic duct orifice $[14,27]$. It is also thought that ES is effective in the prevention of PEP when performing multiple transpapillary procedures during ERCP. Alternatively, MS placement may obstruct the pancreatic duct orifice so much that ES cannot decrease the tension. ES more effectively prevented PEP in patients undergo- 
- Table 7 Risk factors for post-ERCP pancreatitis in patients who underwent intraductal ultrasound.

\begin{tabular}{|c|c|c|c|c|c|}
\hline \multirow[t]{2}{*}{ Variables } & \multicolumn{3}{|c|}{ Univariable analysis ${ }^{1}$} & \multicolumn{2}{|c|}{ Multivariable analysis } \\
\hline & $\begin{array}{l}\text { PEP } \\
n=21\end{array}$ & $\begin{array}{l}\text { No PEP } \\
n=94\end{array}$ & $P$ value $^{1}$ & OR & $P$ value \\
\hline Younger age, $<60$ years & $2(9.5)$ & $10(10.6)$ & 0.87 & & \\
\hline Female sex & $10(47.6)$ & $41(43.6)$ & 0.74 & & \\
\hline Body mass index $>25 \mathrm{~kg} / \mathrm{m}^{2}$ & $3(14.2)$ & $18(19.1)$ & 0.60 & & \\
\hline \multicolumn{6}{|l|}{ Biliary location of tumor } \\
\hline - Papilla & $4(19.0)$ & $17(18.1)$ & 0.67 & & \\
\hline - Hilar bile duct & $2(9.5)$ & $11(11.7)$ & 0.56 & & \\
\hline - Extrahepatic bile duct & $15(71.4)$ & $66(70.2)$ & 0.63 & & \\
\hline Common bile duct diameter $<8 \mathrm{~mm}$ & $6(28.5)$ & $33(35.1)$ & 0.57 & & \\
\hline Periampullary diverticulum & $2(9.5)$ & $5(5.3)$ & 0.47 & & \\
\hline Jaundice at ERCP & $16(76.1)$ & $53(56.4)$ & 0.09 & 1.99 & 0.11 \\
\hline Long procedural time, $>40 \mathrm{~min}$ & $15(71.4)$ & $42(44.7)$ & $0.03^{1}$ & 2.89 & $0.01^{1}$ \\
\hline Cannulation method type ${ }^{2}$ & $2(9.5)$ & $21(22.3)$ & 0.18 & & \\
\hline Non-ES & $18(85.7)$ & $55(58.5)$ & 0.02 & 4.54 & $0.01^{1}$ \\
\hline Transpapillary biliary duct biopsy & $9(42.9)$ & $43(45.7)$ & 0.81 & & \\
\hline Pancreatic duct injection & $15(71.4)$ & $57(60.6)$ & 0.36 & & \\
\hline Double guidewire cannulation & $3(14.2)$ & $9(9.6)$ & 0.52 & & \\
\hline Biliary duct stent placement & $16(76.1)$ & $84(87.5)$ & 0.11 & & \\
\hline Pancreatic duct stent placement & $1(4.76)$ & $1(1.06)$ & 0.33 & & \\
\hline \multicolumn{6}{|c|}{$\begin{array}{l}\text { ERCP, endoscopic retrograde cholangiopancreatography; non-ES, endoscopic sphincterotomy not performed; IDUS, intraductal ultrasound; OR, odds ratio; PEP, } \\
\text { post-ERCP pancreatitis. } \\
\text { The data are shown as } n \text { (\%). } \\
{ }^{1} \text { Univariable analyses were performed using Chi-squared tests. P values of < } 0.05 \text { (bold) were considered significant and included in the logistic regression analysis } \\
{ }^{2} \text { Not guided by wire. }\end{array}$} \\
\hline
\end{tabular}

ing PS placement, and the incidence of PEP tended to be higher in patients with MSs regardless of whether ES was performed.

ES is associated with life-threatening complications, such as bleeding and perforation [21]. Assessment of adverse events in the present study showed that bleeding due to ES was observed in $3 \%$ of patients, although no cases were life-threatening. These observations are consistent with those reported in an earlier study [23]. Several studies also reported that ES before biliary stenting increases the risk of cholangitis in patients with proximal bile duct obstruction [26, 28]. However, in this study, cholangitis was not increased in the ES group and adverse events associated with ES rarely resulted in major clinical issues. ERCP is often repeated to treat stent occlusion and for further examination of biliary neoplasms, and ES is thought to protect against PEP not only following the first ERCP but also following subsequent procedures.

The principal limitations of this study are that the data were collected retrospectively and therefore may be subject to selection and information bias. Due to the small number of patients, some of the differences between patients were not statistically significant. This study reports exploratory analysis, which is an- other limitation. In this study, only MSs with a diameter of 10 $\mathrm{mm}$ were used. All patients with covered MSs underwent ES. Therefore, it was difficult to compare covered and uncovered MSs, as well as MSs with different diameters, in this study.

In addition, because this study assessed the risk factors for PEP after ERCP for biliary neoplasms, we did not exclude cases of exposed ampullary carcinoma, in which ES is difficult. A randomized controlled trial is required to confirm our findings regarding the effect of ES on PEP in patients with biliary neoplasms.

There are two considerations regarding limitations of the statistical analysis in this study. First, propensity score matching, despite its popularity, is problematic and sometimes increases, rather than reduces, bias. Second, variables are selected in this log binomial model using $P$ values. In this study, factors used in multivariable analysis were selected based on the results of univariable analyses, meaning type I errors may have occurred. Therefore, there are limitations regarding the adjustment of bias.

In conclusion, the frequency of PEP was lower in the ES group than in the non-ES group. ES may therefore be a useful 
- Table 8 Risk factors for PEP in the patients who underwent transpapillary bile duct biopsy.

\begin{tabular}{|c|c|c|c|c|c|}
\hline \multirow[t]{2}{*}{ Variables } & \multicolumn{3}{|c|}{ Univariable analysis ${ }^{1}$} & \multicolumn{2}{|c|}{ Multivariable analysis } \\
\hline & $\begin{array}{l}\text { PEP } \\
n=18\end{array}$ & $\begin{array}{l}\text { No PEP } \\
n=67\end{array}$ & $P$ value & $\mathbf{R R}$ & $P$ value \\
\hline Younger age, $<60$ years & $2(11.1)$ & $9(13.3)$ & 0.79 & & \\
\hline Female & $13(72.2)$ & $27(40.3)$ & $0.02^{1}$ & 5.25 & $0.03^{1}$ \\
\hline Body mass index $>25 \mathrm{~kg} / \mathrm{m}^{2}$ & $5(27.8)$ & $17(25.0)$ & 0.84 & & \\
\hline \multicolumn{6}{|l|}{ Biliary location of tumor } \\
\hline - Papilla & $3(16.7)$ & $4(6.0)$ & 0.16 & & \\
\hline - Hilar bile duct & $13(72.2)$ & $51(76.1)$ & 0.76 & & \\
\hline - Extrahepatic bile duct & $2(11.1)$ & $12(17.9)$ & 0.72 & & \\
\hline Common bile duct diameter $<8 \mathrm{~mm}$ & $10(55.6)$ & $20(29.9)$ & $0.04^{1}$ & 1.52 & 0.32 \\
\hline Periampullary diverticulum & $2(11.1)$ & $4(6.0)$ & 0.45 & & \\
\hline Jaundice at ERCP & $12(66.7)$ & $34(50.8)$ & 0.23 & & \\
\hline Long procedural time, $>40 \mathrm{~min}$ & $11(61.1)$ & $33(49.3)$ & 0.37 & & \\
\hline Cannulation method type ${ }^{2}$ & $3(16.7)$ & $13(19.4)$ & 0.79 & & \\
\hline Non-ES & $16(88.9)$ & $35(52.2)$ & $0.005^{1}$ & 5.26 & $0.017^{1}$ \\
\hline IDUS & $9(50.0)$ & $43(64.2)$ & 0.27 & & \\
\hline Pancreatic duct injection & $12(66.7)$ & $39(58.2)$ & 0.52 & & \\
\hline Double guidewire cannulation & $5(27.7)$ & $6(9.0)$ & $0.03^{1}$ & 1.50 & 0.29 \\
\hline Biliary duct stent placement & $14(77.8)$ & $51(76.1)$ & 0.88 & & \\
\hline Pancreatic duct stent placement & $0(0)$ & $4(5.97)$ & 0.57 & & \\
\hline \multicolumn{6}{|c|}{$\begin{array}{l}\text { ERCP, endoscopic retrograde cholangiopancreatography; non-ES, endoscopic sphincterotomy not performed; IDUS, intraductal ultrasound; RR, relative risk; PEP, } \\
\text { post-ERCP pancreatitis. } \\
\text { The data are shown as } n \text { (\%). } \\
{ }^{1} \text { Univariable analyses were performed using Chi-squared tests. P values of < } 0.05 \text { (bold) were considered significant and included in the logistic regression analysis. } \\
{ }^{2} \text { Not guided by wire. }\end{array}$} \\
\hline
\end{tabular}

method for preventing PEP in patients with biliary neoplasm, particularly when performing transpapillary biopsy and IDUS.

\section{Acknowledgments}

We thank all members of the Second Department of Internal Medicine, the Clinical Study Support Center, and the Second Department of Surgery at Wakayama Medical University, the Second Department of Internal Medicine at Osaka Medical College, and the Department of Gastroenterology and Hepatology at Kindai University.

\section{Competing interests}

The authors declare that they have no conflict of interest.

\section{References}

[1] Freeman ML, Nelson DB, Sherman S et al. Complications of endoscopic biliary sphincterotomy. NEJM 1996; 335: 909-918

[2] Freeman ML, DiSario JA, Nelson DB et al. Risk factors for post-ERCP pancreatitis: a prospective multicenter study. Gastrointest Endosc 2001; 54: 425-434

[3] Cheng CL, Sherman S, Watkins JL et al. Risk factors for post-ERCP pancreatitis: a prospective multicenter study. Am J Gastroenterol 2006; 101: 139-147

[4] Testoni PA, Mariani A, Giussan A et al. Risk factors for post-ERCP pancreatitis in high-and low-volume centers and among expert and nonexpert operators: a prospective multicenter study. Am J Gastroenterol 2010; 105: 1753-1761

[5] Freeman ML, Guda NM. Prevention of post-ERCP pancreatitis: a comprehensive review. Gastrointest Endosc 2004; 7: 845-864

[6] Cotton PB, Garrow DA, Gallagher J et al. Risk factors for complications after ERCP: a multivariate analysis of 11,497 procedures over 12 years. Gastrointest Endosc 2009; 70: 80-88

[7] Iorgulescu A, Sandu I, Turcu F et al. Post-ERCP acute pancreatitis and its risk factors. J Med Life 2013; 6: 109-113 
[8] Otsuka T, Kawazoe S, Nakashita S et al. Low-dose rectal diclofenac for prevetion of post-endoscopic retrograde cholangiopancreatography pancreatitis:a randomized controlled trial. J Gastroenterol 2012; 47: 912-917

[9] Choudhary A, Bechtold ML, Arif M et al. Prophylactic temporary 3F pancreatic duct stent to prevent post-ERCP pancreatitis in patients with a difficult biliary cannulation: a multicenter, prospective, randomized study. Gastrointest Endosc 2012; 76: 578-585

[10] Mazaki T, Mado K, Masuda $\mathrm{H}$ et al. Pancreatic stents for prophylaxis against post-ERCP pancreatitis: a meta-analysis and systematic review. Gastrointest Endosc 2011; 73: 275-282

[11] Tse F, Yuan Y, Moayyedi P et al. Guide wire-assisted cannulation for the prevention of post-ERCP pancreatitis: a systematic review and meta-analysis. Endoscopy 2013; 45: 605-618

[12] Sofi AA, Nawras A, Alaradi OH et al. Does endoscopic sphincterotomy reduce the risk of post-endoscopic retrograde cholangiopancreatography pancreatitis after biliary stenting? A systematic review and meta-analysis Dig Endosc 2016; 28: 394-404

[13] Freeman ML. Pancreatic stents for prevention of post-endoscopic retrograde cholangiopancreatography pancreatitis. Clin Gastroenterol Hepatol 2007; 5: 1354-1365

[14] Simmons DT, Petersen BT, Gostout C]. Risk of pancreatitis following endoscopically placed large-bore plastic biliary stents with and without biliary sphincterotomy for management of postoperative bile leaks. Surg Endosc 2008; 22: 1459-1463

[15] Nishikawa T, Tsuyuguchi T, Sakai Y et al. Factors affecting the accuracy of endoscopic transpapillary sampling methods for bile duct cancer. Dig Endosc 2014; 26: 276-281

[16] Yamashita Y, Ueda K, Kawaji Y et al. The wire-grasping method as a new technique for forceps biopsy of biliary strictures: A prospective randomized controlled study of effectiveness. Gut Liver 2016; 10 : 642-648

[17] Cotton PB, Lehman G, Vennes J et al. Endoscopic sphincterotomy complications and their management: an attempt at consensus. Gastrointest Endosc 1991; 37: 383-393
[18] Gum PA, Thamilarasan M, Watanabe J et al. Aspirin use and all-cause mortality among patients being evaluated for known or suspected coronary artery disease: A propensity analysis. JAMA 2001; 286: 1187-1194

[19] Bergstrahl EJ, Konsanke JL. Computerized matching of cases to control. Technical Reports 56 Rochester, MN: Department of Health Science Research, Mayo Clinic; 1995

[20] Luo Z, Gardiner JC, Bradley C] et al. Applying propensity score methods in medical research: pitfalls and prospects. Med Care Res Rev 2010; 67: 528-554

[21] Austin PC. An introduction to propensity score methods for reducing the effects of confounding in observational studies. Multivariate Behav Res 2011; 46: 399-424

[22] Kuss O, Legler T, Börgermann J et al. Treatments effects from randomized trials and propensity score analyses were similar in similar populations in an example from cardiac surgery. J Clin Epidemiol 2011; 64: 1076-1084

[23] Rabenstein T, Schneider HT, Bulling D et al. Analysis of the risk factors associated with endoscopic sphincterotomy techniques: preliminary results of a prospective study, with emphasis on the reduced risk of acute pancreatitis with low-dose anticoagulation treatment. Endoscopy 2000; 32: 10-19

[24] Akashi R, Kiyozumi T, Tanaka T et al. Mechanism of pancreatitis caused by ERCP. Gastrointest Endosc 2002; 55: 50-54

[25] Kawakubo K, Isayama H, Nakai Y et al. Risk factors for pancreatitis following transpapillary self-expandable metal stent placement. Surg Endosc 2012; 26: 771-776

[26] Zhou H, Li L, Zhu F et al. Endoscopic sphincterotomy associated cholangitis in patients receiving proximal biliary self-expanding metal stents. Hepatobiliary Pancreat Dis Int 2012; 11: 643-649

[27] Barthel JS. Internal stents and postsurgical bile leaks. Am J Gastroenterol 1996; 91: 1469-1470

[28] Sung JY, Leung JW, Shaffer EA et al. Ascending infection of the biliary tract after surgical sphincterotomy and biliary stenting. J Gastroenterol Hepatol 1992; 7: 240-245 